\title{
SEXUALIDADE NA TERCEIRA IDADE: COMPREENSÃO E PERCEPÇÃO DO IDOSO, FAMÍLIA E SOCIEDADE
}

Maria Anunciada Souto SANTANA ${ }^{1}$

Elândia Cristina Luna de LUCENA²

Katia Maria Medeiros LIMA ${ }^{3}$

Francisco Assis DANTAS NETO ${ }^{4}$

Maria Cidney da Silva SOARES ${ }^{5}$

${ }^{1}$ Enfermeira Assistencial do Centro Hospitalar João XXIII. E-mail: anunciadass1231@hotmail.com

${ }^{2}$ Enfermeira Assistencial do Hospital D. Pedro I.

${ }^{3}$ Enfermeira Assistencial da Unidade de Pronto Atendimento de Campina Grande PB.

E-mail: katemmlima@hotmail.com

${ }^{4}$ Graduando em Enfermagem pela Faculdade de Ciências Médicas de Campina Grande.

E-mail: franciscodantas_1@hotmail.com

${ }^{5}$ Doutoranda da Universidade Federal da Paraíba. E-mail: profcidneysoares@ hotmail.com

Recebido em: 22/05/2014 - Aprovado em: 30/06/2014 - Disponibilizado em: 30/07/2014

RESUMO - INTRODUÇÃO: O envelhecimento da população mundial é um fenômeno relativamente recente e, em particular no Brasil é um fenômeno social incontestável. OBJETIVO: compreender as percepções do idoso e da família sobre a sexualidade na terceira idade nos dias atuais. METODOLOGIA: A pesquisa se caracteriza como bibliográfica com abordagem qualitativa, a qual foi realizada um levantamento do acervo referente ao tema estudado. Assim, se buscou junto á livros e artigos atualizados o tema abordado. Adotou-se o método qualitativo por expressar os significados de diferentes ideias que propõem uma discursão dos fatos apresentados. RESULTADOS: Após analise da literatura selecionada foi elaborado um texto preliminar para melhor entendimento do estudo, nesta fase foi realizada uma literatura exploratória do marco teórico, selecionado logo em seguida os elementos relevantes ao estudo a fim de vislumbrar o conteúdo da forma global. Foram encontrados dados que trazem com bastante ênfase na sexualidade do idoso onde a família está envolvida nesse processo de convivência com os problemas que surge com o idoso quanto a sua sexualidade. São paradigmas e fatores que são abordados pela sociedade e família junto a estes. Portanto o tema nos faz refletir que idosos, assim como os jovens sentem necessidade do sexo, e não têm como prioridade o ato em si, e sim trata a sexualidade como forma de receber e retribuir afeto e carinho, fazendo com que se sintam capazes de despertar desejos.

Palavras-chave: terceira idade, sexualidade, saúde, família.

ABSTRACT - INTRODUCTION: The aging world population is a relatively recent and , in particular phenomenon in Brazil is an indisputable social phenomenon. OBJECTIVE: To understand the perceptions of the elderly and family about sexuality in old age today. METHODOLOGY : The research literature is characterized as a qualitative approach in which a survey of the acquis concerning the subject studied was performed. Thus, we sought next to the books and articles updated the topic addressed. Adopted a qualitative method for expressing the meanings of different ideas that suggest a discursão the facts presented . RESULTS : The analysis of the selected literature a preliminary text was developed to better understand the study at this stage an exploratory literature on the theoretical framework, selected soon after the elements relevant to the study in order to glimpse the contents of the global shape was performed . Database quite emphatically that bring the sexuality of the elderly where the family is involved in this process of coexistence with the problems that arise with the elderly about his sexuality were found . Are paradigms and factors that are addressed by society and family along to these. So the topic 
makes us think that the elderly, and young people feel they need sex, and have priority as the act itself, but rather treats sexuality as a way to receive and reciprocate affection and care, making them feel able to awakening desires .

Keywords : elderly, sexuality, health, family .

\section{INTRODUÇÃO}

De acordo com o Instituto Brasileiro de Geografia e Estatística, a população idosa está crescendo a uma taxa de oito vezes maior $\mathrm{d}$ que a jovem. Consequentemente essa mudança nítida no perfil populacional traz consigo novas responsabilidades aos gestores e serviços de saúde, onde os mesmos devem estar preparados para atender de maneira satisfatório este grupo populacional. Segundo o Ministério da Saúde, o desafio do Brasil para este século é oferecer suporte de qualidade de vida para uma população com mais de 32 milhões de idosos, na sua maioria de nível socioeconômico e educacional baixo e com alta prevalência de doenças crônicas.

O envelhecimento da população mundial é um fenômeno relativamente recente e, em particular no Brasil é um fenômeno social incontestável. Basta um olhar mais aguçado ao nosso redor para percebermos que o crescimento da população considerada idosa - de 65 anos ou mais - é um fato. Paralelo ao crescimento desta população, que a cada dia adquire maior visibilidade em nossa sociedade, surge várias questões relacionadas a esse novo ator social - o idoso (ALMEIDA; PATRIOTA, 2009).
O tema sexualidade nem sempre é tratado com abertura, pois nos remete a vivencias pessoais extremamente íntimas, especialmente quando falamos sobre sexo na velhice (SILVA, 2008).

Existe ainda na nossa cultura uma falsa ideia de que o (a) velho (a) não tem desejo ou vida sexual. Essa premissa é semelhante à teoria do começo do século, de que a criança não tem sexualidade. Da mesma forma a sociedade tenta negar a sexualidade do idoso. As pessoas acham feio, negam-se a aceitar que o idoso possa querer namorar. Esquecem que a sexualidade não é só genitalidade, existe também uma afetividade que é essencial ao ser humano (GRADIM, et al, 2008).

Com a chegada da velhice, as alterações anatômicas são principalmente as mais visíveis e manifestam-se em primeiro lugar. A pele que resseca, tornando-se mais quebradiça e pálida, perdendo o brilho natural da jovialidade. Os cabelos que embranquecem e caem com maior frequência e facilidade não são mais naturalmente substituídos, principalmente nos homens. O enfraquecimento do tônus muscular e da constituição óssea leva a mudanças na postura do tronco e das pernas, acentuando ainda mais as curvaturas da coluna torácica e lombar. As 
articulações tornam-se mais endurecidas, reduzindo assim a extensão dos movimentos e produzindo alterações no equilíbrio e na marcha. Nas vísceras, produz-se uma alteração causada pelos elementos glandulares do tecido conjuntivo e certa atrofia secundária, como a perda de peso.

As mudanças atingem, simultaneamente, os aspectos institucionais da realidade familiar bem como as identidades pessoais e as relações mais íntimas entre os membros da família. Nesse sentido, Castells (2003) observa que "ao nível dos valores sociais, a sexualidade torna-se uma necessidade pessoal que não deve necessariamente ser canalizada e institucionalizada para o interior da família" (p. 261). A possibilidade de gerar filhos sem o concurso da relação sexual "abre horizontes inteiramente novos à experimentação social" (p. 262).

\section{METODOLOGIA}

Essa pesquisa se caracteriza como bibliográfica com abordagem qualitativa, a qual foi realizado um levantamento do acervo referente ao tema estudado. Assim, se buscou junto á livros e artigos atualizados, compreender as percepções do idoso e da família sobre a sexualidade na terceira idade nos dias atuais.

Quanto a pesquisa bibliográfica, Gil (2002) comenta que a pesquisa bibliográfica é desenvolvida utilizando como fundamental material previamente elaborado, constituindo principalmente no levantamento e recuperação das informações impressas em livros, dissertações de mestrado, monografias e outros.

Também referente a pesquisa bibliográfica, Boaventura (2007) comenta que a pesquisa bibliográfica é desenvolvida utilizando como fundamental material previamente elaborado, constituindo principalmente no levantamento e recuperação das informações impressas em livros, dissertações de mestrado, monografias, artigos científicos e outros.

Adotou-se o método qualitativo por expressar os significados de diferentes ideias que propõem uma discussão dos fatos apresentados. Com isso se percebe uma forte tendência por uma maior utilização dos métodos qualitativos de pesquisa, sobretudo no campo das ciências humanas.

\section{COLETA E ANÁLISE DE DADOS}

Inicialmente foi realizado um levantamento bibliográfico de livros, artigos consensos e guidelines relacionado com a temática estudada. Os artigos e periódicos científicos originais e de revisão ou base de dados incluídos no estudo foram coletados de revista cientifica indexadas tanto impressas quanto on-line. 
Buscou-se realizar com esta coleta uma maior demarcação e seleção da temática estudada, para que seja possível uma ampla compreensão das referencias teóricas. E consequentemente contribuir para outras pesquisas futuras ou que estejam em andamento.

Foi realizada uma busca ativa de publicações bibliográficas relacionadas á temática estudada, esta procura se deu nas bibliotecas anteriormente referidas e para a investigação dos artigos científicos utilizando indexadores e palavras-chaves concernentes, a realização da pesquisa. Através da combinação destas palavras junto com a realização nominal do assunto foi possível relacionar aqueles que possuem relevância para a concretização dos objetivos. Foram utilizados descritores as palavras chaves: Sexualidade, terceira idade, saúde e família. Em seguida foi feita uma leitura de todas as informações coletadas e selecionados os tópicos que foram discutidos baseados na relevância do tema.

Após analise da literatura selecionada foi elaborado um texto preliminar para melhor entendimento do estudo: nesta fase foi realizada uma literatura exploratória do marco teórico, selecionado logo em seguida os elementos relevantes ao estudo a fim de vislumbrar o conteúdo de forma global.

A construção de redação e relatório final do estudo se tornou estruturado com o registro final acerca da compreensão das percepções do idoso e da família sobre a sexualidade na terceira idade nos dias atuais.

\section{RESULTADOS E DISCUSSÃO}

Este estudo, realizado por Maria Anunciada de Souto Santana teve como intuito mostrar aos leitores as percepções do idoso e da família sobre a sexualidade na terceira idade nos dias atuais, demonstrando ainda, quais são fatores sociais que possuem interferência na sexualidade das pessoas de terceira idade.

Mediante o objetivo de compreender as percepções do idoso e da família sobre a sexualidade na terceira idade nos dias atuais, foram elaborados tópicos estruturados para melhor compreensão do leitor quanto a apresentação dos resultados.

\subsection{PARADIGMAS SOCIAIS QUANTO A SEXUALIDADE DO IDOSO}

Um das grandes dificuldades que fundamenta este estereótipo de que a velhice convive com a assexualidade é a ênfase que se atribui para a dimensão sexual. Em consequência disso, tanto para jovens há mais tempo ce como para jovens há menos tempo, ao que parece, vivemos numa ditadura do orgasmo e da frustração, sustentada sobremaneira pela influência midiática. A negação da sexualidade, de manifestações amorosas e a infantilização do idoso 
concorrem para que estes apresentem dificuldades para se tornar mais independentes, bem como para desenvolver sua sexualidade e estabelecer relacionamentos quaisquer que sejam estes (PRIORE, 2006).

Contudo, apesar da abertura social que há para discussão de assuntos desse âmbito, a maioria da população ainda se apresenta constrangida para discutir a respeito desses assuntos, principalmente, quando concernentes a questões relacionadas à sexualidade na Terceira Idade (SANTOS, 2003).

Para algumas pessoas, com a progressão da idade, há uma concomitante anulação desejo sexual para a Terceira Idade, enquanto, para outras, há apenas uma modificação, entretanto, de modo geral, o que se evidencia é que para uns e outros é uma constante e cômoda negação do desejo do idoso pela sociedade. Com essa negação, a sociedade sedimenta e reproduz seus próprios medos e inseguranças, suas preocupações no que diz respeito ao próprio futuros e sua possível incapacidade para amar a medida que envelhecem (RODRIGUES, 2000).

\section{FATORES QUE INTERFEREM NA SEXUALIDADE NA TERCEIRA IDADE}

Com uma visão restrita, tanto em relação à sexualidade quanto à velhice, a sociedade, muitas vezes, classifica este período da vida como um período de assexualidade e até mesmo de androginia. Dessa forma, neste período, o indivíduo teria que unicamente assumir o papel de avô, ou ainda, de avó, ao lhe ser delegado pelos filhos o cuidado de seus netos, na expectativa de que os monitorem enquanto concomitantemente realiza atividades como o tricô e assiste à televisão e usufrui de sua aposentadoria (RISMAN, 2005).

Manter durante toda a vida uma prática sexual contínua e equilibrada. Quanto mais atividade sexual maior a possibilidade de ser manter desta forma, quanto menos sexo menos vontade e a frequência diminui. O equilíbrio no relacionamento sexual quando jovem é mantido com o passar dos anos. O importante é ter uma vida sexual regular. Se para a mulher jovem é importante a prática sexual com regularidade, para a idosa isso é ainda mais necessário, pois seu desempenho sexual depende mais da regularidade que o da mulher jovem ( VASCONCELLOS, 2004).

$\mathrm{Na}$ prática essa atitude equivale a um processo de auto estimulação: pois quanto maior a atividade sexual mais possibilidade de melhorar as condições dos órgãos afetados pelo envelhecimento e, em consequência, maior disposição e interesse em prosseguir com a atividade sexual. 


\section{Aspectos culturais e psicológicos}

Vivemos em um país onde a cultura transmitida pela população mais antiga ainda está muito presente. Primeiramente, as crenças perpassadas nos remetem à análise o que acontece com as pessoas durante a gestação.

Belotti (1975, p. 17) aponta algumas crendices que estavam e podem estar ainda presentes no seio da sociedade:

“[...] o ventre mais pontudo da mãe durante a gravidez é sinal que vai nascer menino, ao passo que um ventre mais chato, largo, distendido, indica que vai nascer menina... se a pulsação cardíaca do feto for rápida, será um garoto; se lenta menina... enfiando uma moeda nas costas, sob a roupa da mãe, se ela cair por terra com a „,cara ${ }^{\text {ee }}$ para cima nascerá um menino, caso contrário, menina."

A vivência dos idosos mostra ainda, em algumas situações, como a aceitação da infidelidade masculina, está presente na cultura de suas vidas, embora consigam, principalmente reunidos no grupo, desmistificar e avançar em alguns aspectos que até então eram vistos e tidos como errados. Podemos citar como exemplo as mulheres que admitem sentir a necessidade de estar usufruindo sua sexualidade e do sexo propriamente dito. Também podemos mencionar que as pessoas, hoje, de terceira idade, conseguem conversar com mais liberdade e menos constrangimentos com seus filhos (as) e netos (as) sobre sexo. Salientamos, aqui, a presença forte que há no amor e nos relacionamentos afetivos ocorridos dentro do grupo (CAPODIECI, 2000)

\section{Aspectos físicos}

Cada pessoa possui caracteres especiais e particulares. Se exames forem realizados em milhões de pessoas, no mundo inteiro, certamente será percebido que, embora fisicamente semelhantes, seus gens e células são diferentes. Durante o processo de envelhecimento humano, muitos são os fatores que determinam algumas características biológicas ou comportamentais da pessoa humana.

Assim, para Oliveira (2002, p. 21):

Esse ser particular vai desenvolvendo a sua personalidade através de diversos "bombardeios", mensagens, acontecimentos, que vão sendo codificados, armazenados e vividos ao longo deste desenvolvimento, no cotidiano das relações do homem com a natureza, com outros homens e com Deus.

O autor faz referência ao comportamento do ser humano. Cada pessoa tem sua composição genética definida ao 
nascer, e esta o acompanhará pelo resto de sua vida. Pelas características herdadas, os seres humanos poderão desenvolver patologias que necessitarão de acompanhamento de profissionais especializados. Outros fatores, que poderão influenciar no processo de envelhecimento, são as relações que a pessoa estabelece em seu meio; suas relações sociais que interferem em sua vida. Alguns problemas de ordem física podem influenciar no relacionamento sexual dos casais que estão envelhecendo.

Geralmente, o comportamento da pessoa jovem ou adulta terá suas consequências mais significativas na velhice, ou seja, quando o corpo físico se encontra mais debilitado pelo tempo. É comum vermos, na sociedade dita moderna e avançada, jovens preocupados somente com o presente. E é nesse "embalo" que usufruem de bebidas alcoólicas, fumo, comidas gordurosas, doces em excesso, têm noites mal dormidas, etc. que, no futuro, comprometerão seu desempenho físico, mental e emocional.

De acordo com o (OLIVEIRA, 2002), ao chegar à terceira idade, homens e mulheres buscam incansavelmente reverter o que o tempo lhes trouxe, ou não, através de seus excessos. E, nesse sentido, podemos constatar alguns problemas físicos que dificultam ou impossibilitam a sexualidade das pessoas de terceira idade. Ao chegar à terceira idade, homens e mulheres buscam incansavelmente reverter o que o tempo lhes trouxe, ou não, através de seus excessos. E, nesse sentido, podemos constatar alguns problemas físicos que dificultam ou impossibilitam a sexualidade das pessoas de terceira idade (OLIVEIRA, 2002).

\section{COMPREENSÃO DA SEXUALIDADE PELOS IDOSOS JUNTO AOS SEUS FAMILIARES}

Os idosos sofrem de problemas sexuais e preocupações que não são diferentes daqueles das pessoas jovens; todavia os fatores biológicos e psicológicos podem exigir mais atenção. Os idosos contemporâneos, e, sobretudo, os casais idosos, podem ter os mesmos problemas que envolvem as pessoas de todas as idades, daí surgirem certas dúvidas em pessoas mais jovens, preocupadas com que o futuro lhes reserva (BYSTRONSKI, 1995).

Em estudo realizado com idosos no Município de Palmas (PR) por Catusso (p. 34, 2005) com membros do Grupo de Convivência “Anos Dourados”. Demonstrarse-á, através de gráfico, as falas dos sujeitos e a análise dos dados a partir da categoria, influência da família.

- "Eu tenho meu companheiro, mas conheço idosos que não podem nem 
sair de casa, muito menos namorar"... (Suj 02).

- "os netos não achão bom, os filhos também não, então a gente não procura" (Suj. 01)

\section{- $\quad$ "Ah sim, mas a família} não quer.” (Suj. 10).

$\mathrm{O}$ relato acima faz referência à dependência que se formou, em torno da pessoa de terceira idade e de sua família. Em sua literatura, comprova que os adultos são um dos principais fatores que atuam na repressão da sexualidade das pessoas de terceira idade. E é nesse sentido que a pesquisa demonstrou o quanto ainda os idosos estão sujeitos e submissos à opinião de seus familiares, principalmente quando estes residem sob o mesmo teto (FERICGLA, 1992).

O controle das ações, das atividades e até mesmo dos relacionamentos afetivos estão sob o olhar da família que subjuga os sentimentos dos seus idosos. A sexualidade da pessoa de terceira idade torna-se reprimida, uma vez que, a família residente na mesma casa, composta por pessoas que vão além do casal, impede com frequência a privacidade. Dificilmente os cônjuges conseguirão exprimir os sentimentos de maneira desejada, pois o convívio com as demais pessoas pode criar um ambiente onde não há liberdade para expressar os afetos (ERBOLATO, 2000).

\section{CONSIDERAÇÕES FINAIS}

O presente trabalho teve por finalidade possibilitar ao leitor de maneira esclarecedora, um pouco sobre sexualidade na terceira idade, um assunto ainda que ignorado pela sociedade a qual vivemos.

Muitos idosos abatem-se com preconceitos vindos de suas famílias, muita delas chocam-se com a ideia de que estes ainda sentem prazeres, trocam carícias e cometem o ato sexual, ridicularizando-os e fazendo com que este desejo adormeça entre eles.

Atualmente, a ciência possibilita que os idosos tenham uma vida sexual ativa de forma prazerosa. Junto a essa evolução da tecnologia, é necessário que a mentalidade do ser humano também evolua e aceite que o sexo é uma prática normal também para quem não é jovem. Portanto, espera-se uma melhor aceitação da sexualidade na terceira idade, principalmente com maior naturalidade, uma vez que faz parte da saúde e bem estar do idoso.

Concluí que é possível manter viva a sexualidade em nós durante toda a nossa trajetória, principalmente na velhice. Isso se no anteceder de nossas vidas tratamos o 
sexo de maneira positiva e sem traumas. $\mathrm{O}$ tema nos faz refletir que idosos, assim como os jovens sentem necessidade do sexo, e não têm como prioridade o ato em

\section{REFERÊNCIAS}

ALMEIDA, LA; PATRIOTA, LM. Sexualidade na terceira idade: um estudo com idosas usuárias do programa saúde da família do bairro das Cidades - campina grande/PB. QUALITS - Revista eletrônica, ISSN 16774280. Vol.8. No 1 (2009). Disponível em:

http://revista.uepb.edu.br, acesso em: 18 de março de 2012.

BELOTTI, Eliana Gianini. Educar para a submissão. O descondicionamento da mulher. 2. ed. Petrópolis: Vozes, 1975.

BOAVENTURA, E. M. Metodologia da pesquisa: monografia, dissertação, tese. 1 . Ed. São Paulo. Atlas, 2007.

BYSTRONSKI, B. Teorias e Processos Psicossociais da Intimidade Interpessoal. In: RODRIGUES, A (Org.). Psicologia Social para principiantes: estudo da interação humana. Petrópolis: Vozes, 1995.

CALDAS, C.P. "O idoso em seu processo demencial: o impacto na família.” In: MINAYO, M.C.S \& COIMBRA JR., C. (org.). Antropologia, Saúde e Envelhecimento. Rio de Janeiro: Editora Fiocruz, 2002.

CAPODIECI, Salvatore. A idade dos sentimentos: amor e sexualidade após os 60 anos. Tradução de Antonio Angonese. Bauru, São Paulo: EDUSC, 2000.

CASTELLS, M. potere delle identitá. Milano:

Universitá Bocconi Editore, 2003.

CATUSSO, MC. Rompendo o silencio: desvelando a sexualidade em idosos. Revista Virtual Textos \& Contextos. $\mathrm{N}^{\circ} 4$, ano IV, dez. 2005. Disponível em: si, e sim trata a sexualidade como forma de receber e retribuir afeto e carinho, fazendo com que se sintam capazes de despertar desejos. http://revistaseletronicas.pucrs.br. Acesso em: 23/04/2012.

ERBOLATO, Regina M. P. Gostando de si mesmo: a auto-estima. In NERI, Anita Liberelasso. Envelhecer num país de jovens. Significados de velho e velhice. Segundo brasileiros e não idosos. Campinas: Unicamp, 2000.

FERICGLA, Josep M. Sexo y afectividad em la cultura de la ancinidad. In: Envejecer. Una antropologia de la ancinidad. Barcelona: Antropos, 1992.

GOLDANI, A. M. Mulheres e envelhecimento: desafios para novos contratos intergeracionais e de gênero. In: CAMARANO, A. A. muito além dos sessenta anos. Rio de Janeiro: IPEA, 1999.

GRADIM, CVC; SOUSA; AMM, LOBO; JM. A prática sexual e o envelhecimento. Ver. Cogitare Enfermagem. 2007, v. 12(2): p.204-13.

Disponível em: $<$ http://ojs.c3sl.ufpr.br/ojs2/index.php/cogitare/arti cle/view/9820/6726> Acesso em: 26 fev. 2008.

LEITE, R. C. B. O. O idoso dependente em domicílio. Faculdade de Enfermagem. OLIVEIRA, Jairo da Luz. A vida cotidiana do idoso morador de rua: as estratégias de sobrevivência da infância à velhice - Um círculo da pobreza a ser rompido. Canoas: Ed. ULBRA, 2002.

PRIORE, M. D. História do Amor no Brasil. São Paulo: Contexto, 2006.

RISMAN, A. Sexualidade e terceira Idade: Uma visão histórico-cultural. Textos sobre Envelhecimento. Rio de Janeiro, v.8, n.1, 2005.

RODRIGUES, A. et al. Memórias de cuidados na infância, estilo de vinculação e qualidade da relação com pessoas significativas: Estudo com 
grávidas adolescentes. Análise Psicológica.

Lisboa, v. 2, n. 4, p. 643-665, out. 2004.

SALGADO, C. D. S. Mulher idosa: a

feminização da velhice. In: Estudos

interdisciplinares

SANTOS, S. S. Sexualidade e amor na velhice.

Porto Alegre: Sulina, 2003.

SBRASH - REVISTA BRASILEIRA DE

SEXUALIDADE HUMANA. 1998, v9(1)p.19-

21Disponívelem:www.adolec.br/bvs/adolec/P/pdf/

volumes/volume9_1.pdf>

SILVA, RCR; REZENDE, RM, COTTA; LG, ET

AL. O papel da enfermagem na sexualidade da

$3^{\circ}$ idade: informar para prevenir. Rev.

Científica da Família. 2007, v.3: p57. Disponível

em:

<http://www.faminas.edu.br/muriae/editora/enic3/

CBS-057.pdf> Acesso em: 03 mar. 2008.

SILVA, RMO. A sexualidade no envelhecer: um estado com idosos em reabilitação. Acta

Fisiátrica. v. 10: p107-112. São Paulo. 2003.

sobre envelhecimento. UFRGS. V.4. Porto

Alegre: Núcleos de Estudos Interdisciplinares

Universidade Federal da Bahia. Bahia: dissertação

de mestrado, 1995.

VAZ, RA; NODIN, N. A importância do exercício físico nos anos maduros da

sexualidade. Analise psicológica. 2005 v.3, p

329-39. Disponível em:

$<$ http://www.scielo.oces.mctes.pt/pdf/aps/v23n3/v

23n3a11.pdf> Acesso em 10 mar. 2008

ZIMERMAN, Guite I. Velhice: Aspectos

Biopsicossociais. Editora Artes Médicas Sul,

Porto Alegre, 2000. 